\title{
A Prospective Evaluation of Two Rapid Phenotypical Antimicrobial Susceptibility Technologies for the Diagnostic Stewardship of Sepsis
}

\author{
Cesira Giordano $\mathbb{D}$, Elena Piccoli, Veronica Brucculeri, and Simona Barnini \\ SD Ospedaliera di Microbiologia, Azienda Ospedaliero-Universitaria Pisana, Via Paradisa, No. 2, Ed. 200, 56124 Pisa, Italy \\ Correspondence should be addressed to Cesira Giordano; cesira.giordano@gmail.com
}

Received 29 December 2017; Accepted 29 March 2018; Published 10 May 2018

Academic Editor: Klaus P. Hunfeld

Copyright (C) 2018 Cesira Giordano et al. This is an open access article distributed under the Creative Commons Attribution License, which permits unrestricted use, distribution, and reproduction in any medium, provided the original work is properly cited.

Rapid identification of bloodstream pathogens by MALDI-TOF MS and the recently introduced rapid antimicrobial susceptibility testing (rAST) directly from positive blood cultures allow clinicians to promptly achieve a targeted therapy, especially for multidrug resistant microorganisms. In the present study, we propose a comparison between phenotypical rASTs performed in light-scattering technology (Alfred 60AST, Alifax ${ }^{\circledR}$ ) and fluorescence in situ hybridization (Pheno ${ }^{\mathrm{TM}}$, Accelerate) directly from positive blood cultures, providing results in 4-7 hours. Blood samples from 67 patients admitted to the Azienda Ospedaliero-Universitaria Pisana were analyzed. After the direct MALDI-TOF MS identification, the rAST was performed at the same time both on Alfred 60AST and Pheno. Alfred 60AST provided qualitative results, interpreted in terms of clinical categories (SIR). Pheno provided identification and MIC values for each antibiotic tested. Results were compared to the broth microdilution assay (SensiTitre ${ }^{\mathrm{TM}}$, Thermo Fisher Scientific), according to EUCAST rules. Using Alfred 60AST, an agreement was reached, 91.1\% for Gram-negative and 95.7\% for Gram-positive bacteria, while using Pheno, the agreement was $90.6 \%$ for Gram-negative and 100\% for Gram-positive bacteria. Both methods provided reliable results; Alfred 60AST combined with MALDI-TOF MS proved itself faster and cheaper. Pheno provided identification and MIC determination in a single test and, although more expensive, may be useful whenever MIC value is necessary and where MALDI-TOF MS is not present.

\section{Introduction}

Sepsis is defined as life-threatening organ dysfunction caused by a dysregulated host response to infection and is the leading cause of death in intensive care units $[1,2]$. It can cause longterm disability, prolonged hospitalizations, large additional costs for healthcare systems, and loss of quality of life for patients and their families [3]. Empirical antibiotic therapy administered within the first hour of clinical suspicion of sepsis decreases mortality rate but may have multiple negative effects related either to drug side effects or to the rise of multidrug resistance pathogens $[4,5]$. Bacterial multidrug resistance is indeed emerging worldwide at an alarming rate and is recognized now as a major public health threat [6]. It is clear that the actual condition will not be resolved by the development of new antibiotics, as only few molecules have been recently introduced for humans [7, 8]. Therefore, in order to reduce selective pressure on microorganisms and effectively cure septic patients, an antimicrobial susceptibility profile is required as soon as possible. This will allow us to properly address the therapy, avoiding unnecessary antibiotic administrations. Thus, implementing strategies to preserve the activity of existing antimicrobial agents has become a healthcare priority, leading to the development of several antimicrobial stewardship programs $[4,5]$.

Several diagnostic systems have been developed for rapid identification of microorganisms found in positive blood cultures, providing quicker results than conventional methods [9]. In routine clinical practice, laboratory identification (ID) and antimicrobial susceptibility testing (AST) are based primarily on bacterial cultures and are usually completed in 2 days or more, during which time empirical therapy is initiated, based on the suspected causative organism and local epidemiology [10]. The use of new technologies 
able to rapidly detect antimicrobial resistance in bacterial isolates therefore has the potential to reduce the duration of empirical treatment and facilitate the early initiation of targeted therapy. Conventional AST systems indirectly sense change in bacterial population by measuring optical density and require 8-20 hours to obtain results, depending on the microorganism [11]. To date, various methods have been developed to improve conventional AST systems, the socalled rAST [12-17].

In this study, we provide a prospective evaluation of two phenotypical rASTs during sepsis: the Accelerate Pheno (Accelerate Diagnostics, USA) and Alfred 60AST (Alifax SpA, Italy) systems, compared to conventional culture-based identification and broth microdilution assay AST. Pheno is a fully automated system capable of performing identification and AST directly from positive blood cultures within approximately 7 hours. The system relies on gel electrofiltration and fluorescence in situ hybridization for bacterial identification, as well as automated digital microscopy for analyzing bacterial growth rates and for extrapolating MIC values. The Alfred 60AST system is based on a light-scattering technique that reliably detects microbial growth in fluid samples, providing real-time growth curves and bacterial counts $(\mathrm{CFU} / \mathrm{ml})$. The instrument allows only for detection of live bacteria, because the initial blank value reading eliminates inert materials. The system was previously evaluated for microorganism enrichment, determination of microbial count, and diagnosis of central venous catheter-related bloodstream infections [18, 19]. Alfred 60AST was initially conceived for urine screening and for AST of bacterial isolates from urine [20]. Then, it was adapted to rapid AST from positive monomicrobial blood cultures, coupled with a rapid direct identification using MALDI-TOF [21, 22]. AST results are available in 4-6 hours: in the presence of a specific drug, absence of growth is interpreted as sensitivity and growth as resistance to the antibiotic, in terms of clinical categories.

\section{Materials and Methods}

2.1. Blood Samples. Blood samples from 67 patients admitted to the Azienda Ospedaliero-Universitaria Pisana (Pisa, Italy) in the March-August 2017 period were inoculated into blood culture (BC) bottles (Becton, Dickinson \& Co., Milan, Italy), collected at the SD Ospedaliera di Microbiologia and transferred to the Bactec FX instrument (Becton Dickinson) for monitoring bacterial growth. For each patient, two inclusion criteria were followed: (1) we chose the first positive BC, which was apparently monomicrobial at the Gram staining; (2) for Gram-positive cocci seen in grape-like clusters, we chose only BCs that had a time to positivity under 10 hours. Blood cultures containing Gram-negative bacilli from 55 patients and Gram-positive cocci from 12 patients were investigated. After subculture on blood agar plates (bioMérieux, Marcy l'Étoile, France), 5 of 55 (9\%) and 2 of 12 (17\%) cultures were found to be polymicrobial, and ID results were analyzed separately (see Section 3.3). Samples were taken as remnants of standard patient care and used anonymously. For this type of study, no written informed consent was necessary. Microbiology laboratory operation time is weekdays from 8 a.m. to 8 p.m. and weekends from 8 a.m. to 2 p.m. Blood cultures are processed every day during operating hours as soon as they are flagged positive.

2.2. Identification and AST of Bacteria by the Routine Method. From BCs found positive with the Bactec FX instrument (Becton Dickinson), Gram-staining and subcultures onto appropriate solid media-nutrients (e.g., blood agar) and selective plates (MacConkey and Mannitol Salt agar)-were performed, and then plates were incubated overnight at $37^{\circ} \mathrm{C}$. Isolated colonies were identified using MALDI-TOF MS (Bruker Daltonics) and AST was performed with the SensiTitre system (Thermo Fisher Scientific, MA, USA), according to the manufacturer's instructions. Two different SensiTitre plates were used: ITGNEGF for Gram-negatives and ITGPOSF for Gram-positives. Colonies were dissolved in sterile water and the suspension was adjusted to $0.5 \mathrm{McFarland}$ standard, using the Densimat densitometer (bioMérieux). For Staphylococcus aureus and Enterococcus spp. $50 \mu \mathrm{L}$ of the suspension was transferred into $11 \mathrm{~mL}$ Cation-Adjusted Mueller-Hinton Broth (CAMHB) (Thermo Fisher Scientific) and $100 \mu \mathrm{L}$ was inoculated into each well on the SensiTitre plate. For Gram-negatives $10 \mu \mathrm{L}$ was transferred into $11 \mathrm{~mL}$ CAMHB and $50 \mu \mathrm{L}$ of the suspension was inoculated into SensiTitre plate. Plates were incubated for 20-24 hours at $35^{\circ} \mathrm{C}$ in the ARIS ${ }^{\mathrm{TM}}$ Instrument (Thermo Fisher Scientific). The ID and AST results by the routine method were used as reference for evaluation of results obtained by MALDITOF MS and Pheno rapid ID, and Alfred 60AST and Pheno preliminary ASTs. Occasionally, the MIC test strip (Liofilchem, Roseto degli Abruzzi, Italy) was performed in case of ambiguous results.

\subsection{Rapid Identification of Bacteria from Positive Blood} Cultures. For the rapid identification method, herein referred to as direct ID according to a previous paper from Barnini and colleagues [22], a modified protocol was used. Briefly, an $8 \mathrm{ml}$ sample of a positive BC at the Bactec FX was transferred into Serum Separator Tubes (BD Vacutainer systems). Next, bacteria were stratified on the surface of the silicon layer by centrifugation at $3500 \mathrm{rpm}$ for $10 \mathrm{~min}$. The bacterial pellet was entirely collected and suspended in $1 \mathrm{ml}$ of distilled water into an eppendorf tube. Two centrifugations at 13,000 rpm for $2 \mathrm{~min}$, alternated by adding $1 \mathrm{ml}$ of distilled water, were performed, and then the bacterial pellet was allowed to dry at room temperature. If necessary, a further washing step was added in order to eliminate interfering erythrocytes. Next, bacteria were transferred with a wooden stick onto the polished steel target plate for MALDI-TOF MS identification. Gram-positive cocci were further exposed to a protein extraction protocol. In detail, Gram-positive cocci were overlaid with $1 \mu \mathrm{l}$ of absolute ethanol (Fluka, St. Louis, MO, USA). When dry, $1 \mu \mathrm{l}$ of formic acid $(70 \% \mathrm{v} / \mathrm{v}$; Fluka) was added and, when air-dried, $1 \mu \mathrm{l}$ of acetonitrile (Carlo Erba, Milan, Italy) was added. The preparation was overlaid with $1 \mu \mathrm{l}$ of saturated alpha-cyano-4-hydroxycinnamic acid (HCCA) in $50 \%$ acetonitrile and $2.5 \%$ trifluoroacetic acid matrix solution (Bruker Daltonics) and air-dried, thus allowing the crystallization of HCCA with the sample. MALDI-TOF MS 
analysis was performed using a Microflex LT system table top mass spectrometer following the manufacturer's instructions. Captured spectra were analyzed as reported elsewhere [22].

2.4. Rapid AST of Bacteria Using Pheno. Two Pheno system modules were available during our study period, allowing us to test two samples simultaneously. Quality controls were performed in advance, according to the manufacturer's instructions. As soon as the BC bottles were flagged positive, the Gram staining and the direct identification by MALDITOF MS were performed. Then, the Pheno Test BC kits (Accelerate Diagnostics) were run on the Pheno system modules. According to the company, it is recommended to apply the Pheno system within 8 hours after the BC is flagged positive. However, due to our working hours, we extended this period and included samples positive within 16 hours. One $\mathrm{ml}$ of the positive $\mathrm{BC}$ was transferred into the sample vial (Accelerate Diagnostics). The test system was then started, lasting approximately $5 \mathrm{~min}$. Blood culture aliquots $(500 \mu \mathrm{l})$ were also stored at $-80^{\circ} \mathrm{C}$ in order to evaluate eventual discrepancies. The analysis software, Accelerate Diagnostics Host applications, version 1.2.0.87, automatically generated first an ID report and subsequently an AST report. Pheno identified the following microorganisms: Escherichia coli, Klebsiella spp., Enterobacter spp., Proteus spp., Citrobacter spp., Serratia marcescens, Acinetobacter baumannii, and Pseudomonas aeruginosa among Gram-negatives and Staphylococcus aureus, Coagulase-Negative Staphylococci (CoNS), Staphylococcus lugdunensis, Enterococcus faecalis, Enterococcus faecium, Streptococcus spp., Streptococcus pneumoniae, and Streptococcus agalactiae, among Gram-positives. AST was performed as reported in Tables 1(a) and 1(b).

2.5. Rapid AST of Bacteria Using Alfred 60AST. Direct AST of bacteria by Alfred 60AST (Alifax) was carried out by transferring $10 \mu \mathrm{l}$ of broth from positive $\mathrm{BC}$ into a vial containing $3 \mathrm{ml}$ of HB\&L enrichment broth (Alifax). Vials were loaded in the thermostatic area of the Alfred 60AST system, for monitoring bacterial growth up to $0.5 \mathrm{McF}$ arland. Next, the instrument automatically transferred the sample into an AST-Empty vial (Alifax), placed in the refrigerated area. Lyophilized antibiotics (Alifax) were dissolved in $2 \mathrm{ml}$ regenerating solution (provided with the antibiotics) and stored at $4^{\circ} \mathrm{C}$ for up to 6 days (3 days for meropenem). Regenerated antibiotics were loaded into the refrigerated area. The antibiotics tested were CE approved. Four panels were established, based on the antibiotics available, named as follows: Enterobacteriaceae, Gram-negative nonfermenters, S. aureus, Enterococcus. Enterobacteriaceae panel included 10 antibiotics: ceftazidime, cefotaxime, ceftriaxone, cefuroxime, piperacillin/tazobactam, meropenem, gentamicin, levofloxacin, colistin, and trimethoprim-sulfamethoxazole. Gramnegative nonfermenters panel included ceftazidime, amikacin, levofloxacin, gentamicin, and colistin. The $S$. aureus panel comprised cefoxitin, linezolid, teicoplanin, vancomycin, daptomycin, and clindamycin. The Enterococcus panel comprised ampicillin, linezolid, teicoplanin, vancomycin, and daptomycin. The vials dedicated to the AST analysis, each containing $2 \mathrm{ml}$ of HB\&L broth, were stored in the thermostatic area at $37^{\circ} \mathrm{C}$ and automatically loaded with the bacterial suspension $(100 \mu \mathrm{l})$, until $0.5 \mathrm{McF}$ arland was reached, and with the selected antibiotic $(200 \mu \mathrm{l})$. One reference vial was loaded with $100 \mu \mathrm{l}$ of bacterial suspension only and used as a positive control for bacterial growth. Three dedicated algorithms, based on fast growing bacteria (e.g., Klebsiella pneumoniae), medium-slow growing bacteria (e.g., Staphylococcus aureus), and slow growing bacteria (e.g., Acinetobacter baumannii), automatically calculated bacterial growth in the presence of each antibiotic, comparing the growth rate with the reference vial. The majority of the results were available within 3 hours, except for meropenem, piperacillin/tazobactam, teicoplanin, and vancomycin, for which 5 hours were required. Absence of growth was interpreted as sensitivity and growth as resistance to the antibiotic, in terms of clinical categories.

2.6. Data Analysis. The Pheno system identification results were compared with results from the direct identification and from culture-based identification, as routine procedure. The IDs were considered correct if the microorganism produced the same result as in the routine procedure, with no additional microorganisms detected. Polymicrobial cultures were, therefore, excluded from the analysis. The two rapid AST results were compared to the SensiTitre AST, interpreted according to EUCAST guidelines. The categorical agreement (CA) was determined for both methodologies, and the discrepant results were branded according to the current ISO 20776-2 guidelines as follows: very major errors (false susceptibility), major errors (false resistance), or minor errors (intermediate versus resistant or susceptible). The rate of very major errors (VMEs) was calculated dividing the number of false susceptible by the number of resistant strains tested and multiplied by 100, while the rate of major errors (MEs) was calculated dividing the number of false resistance by the number of susceptible strains tested and multiplied by 100 . The rate of minor errors (MiEs) was calculated dividing the number of minor discrepancies by the total number of strains tested and multiplied by 100 . The essential agreement (EA) that is the proportion of total test results within one doubling dilution of the reference result was calculated solely for Pheno system, since Alfred 60AST does not provide MIC values. Statistical analysis was performed using the paired sample $t$ test. The level of significance was set at $p<0.05$.

\section{Results and Discussion}

3.1. The Hospital. The Azienda Ospedaliero-Universitaria Pisana is a tertiary-care university hospital, owning 1.300 beds and accounting for about 50.000 hospitalized patients each year. In 2017 (January-August), the microbiology laboratory processed 3630 positive blood cultures, which represented almost $14 \%$ of total BCs arrived. Of these, 95\% were positive for bacteria and 5\% for fungi. As showed in Figure 1(a), among Gram-positives, CoNS represented $41 \%$ of bacterial isolates, followed by Enterococcus spp. (6\%) and S. aureus (5\%). Common contaminants isolated included Propionibacterium spp. (4\%), Corynebacterium spp. (1\%), and Micrococcus spp. $(<1 \%)$. Among Gram-negatives, K. pneumoniae was the principal microorganism isolated (11\%), followed by 

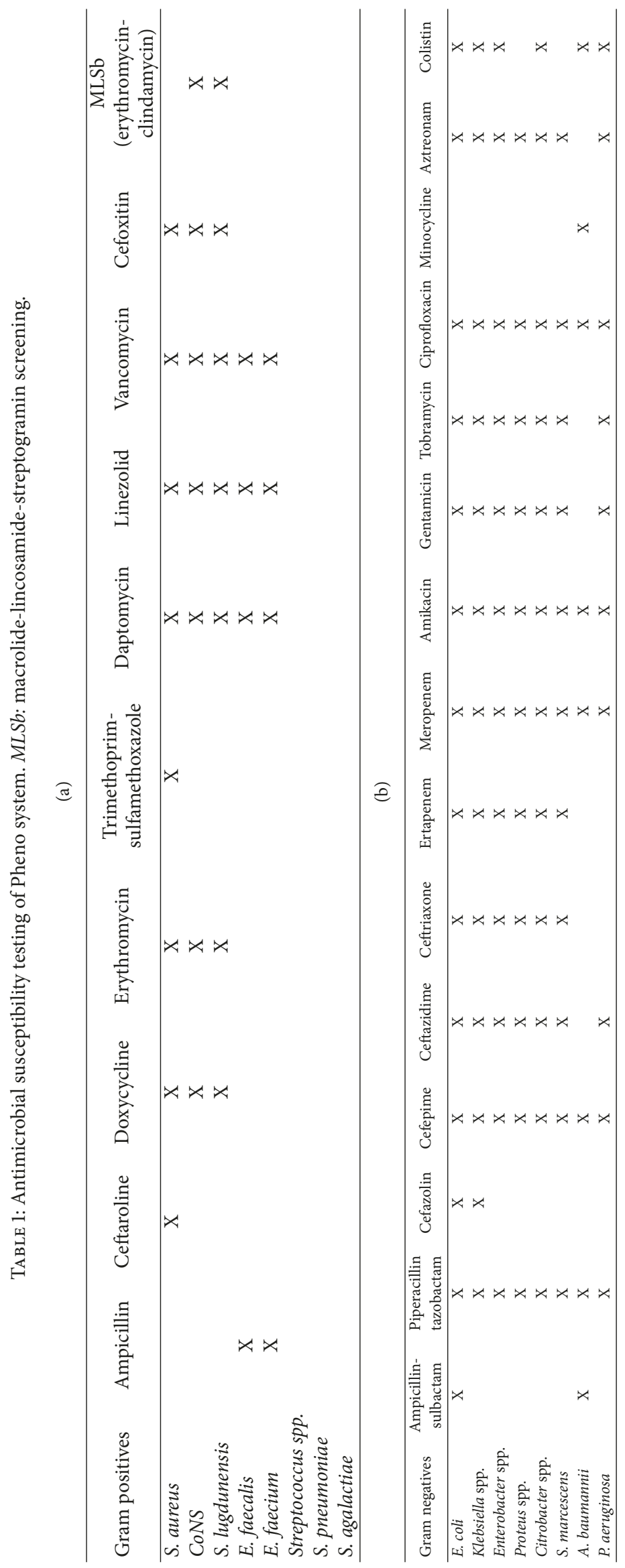


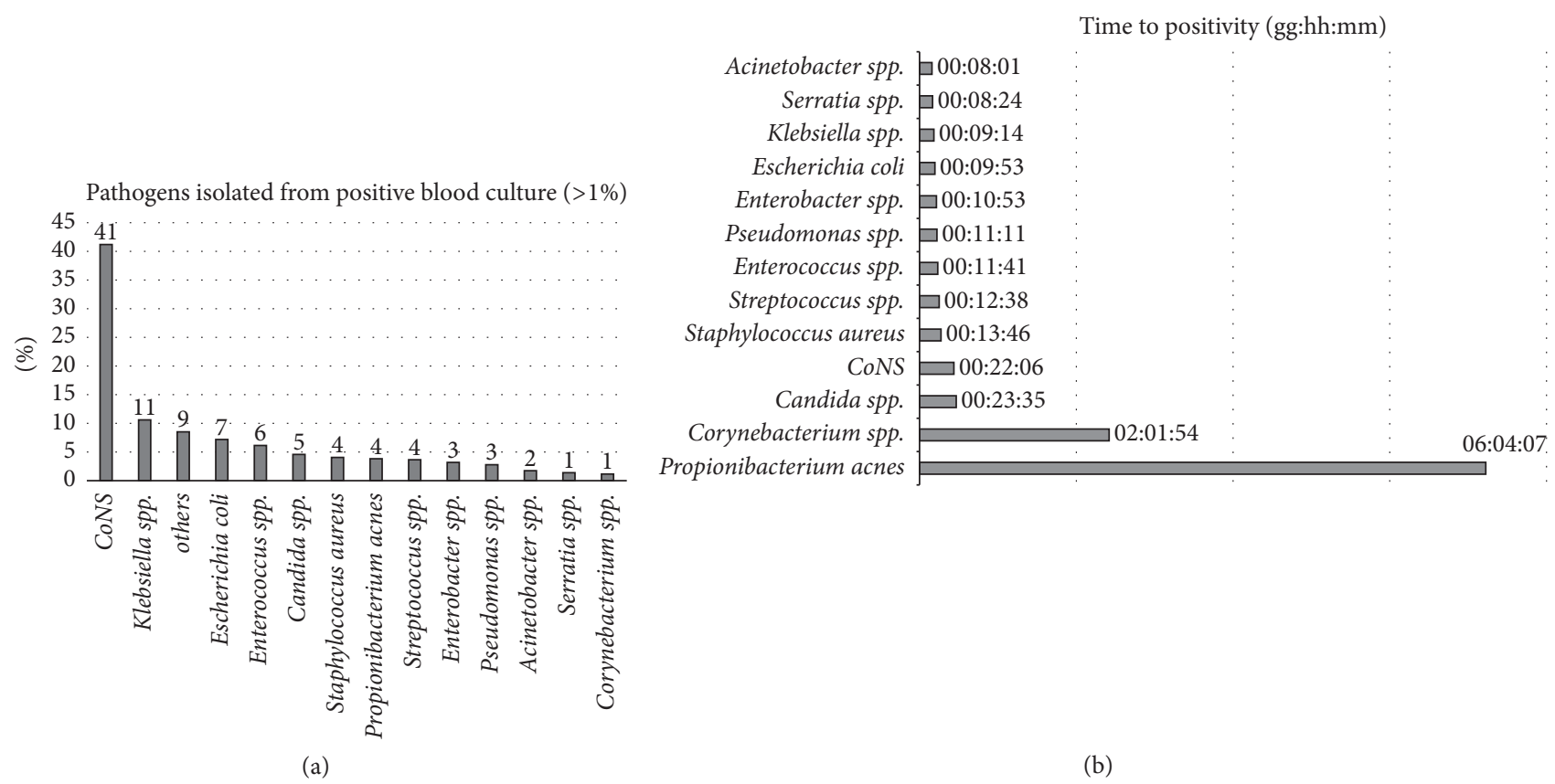

FIGURE 1: The 13 most frequently isolated microorganisms (>1\%) from positive blood cultures in our laboratory during January-August 2017 (a). Median time to positivity of microorganisms in blood cultures (b).

E. coli (7\%). For each patient, the first apparently monomicrobial BC positive for Gram-negatives was included in the study. Since CoNS are the main microorganisms isolated among Gram-positives but often represent a contamination in positive BCs, in order to exclude these microorganisms a further criterion of selection was adopted: the time to positivity of BCs. The median time to positivity (Figure 1(b)) for CoNS was 22 hours, while for $S$. aureus it was appreciably lower (13 hours); for this reason, for Gram-positive cocci in grape-like clusters, only BCs positive within 10 hours were included in the study. Several studies have been considering the prognostic value of time to positivity of BCs, as well as the cogency of this value for diagnosing catheter-related bloodstream infections [23-25]. We observed this trend since $2015[26,27]$ and, in our routine practice, we consider time to positivity as a predictor for $S$. aureus BSI.

3.2. Microorganisms Included in the Study. The performances of Pheno and Alfred 60AST systems were evaluated on a total of 67 positive BCs (Table 2); of these, 60 were monomicrobial and 7 polymicrobial. The following Gramnegative and Gram-positive monomicrobial infections were included in the comparative analysis: 50 caused by Gramnegatives, specifically Acinetobacter baumannii $(n=1)$, Citrobacter koseri $(n=3)$, Enterobacter cloacae $(n=4)$, Escherichia coli $(n=19)$, Klebsiella pneumoniae $(n=19)$, Proteus mirabilis $(n=1)$, Pseudomonas aeruginosa $(n=1)$, and Serratia marcescens $(n=2)$, and 10 caused by Grampositives, precisely Enterococcus faecalis $(n=1)$, Enterococcus faecium $(n=3)$, Staphylococcus aureus $(n=4)$, Streptococcus pneumoniae $(n=1)$, and Streptococcus agalactiae $(n=$ 1). Antimicrobial susceptibility results were produced for 74 isolates, with a total of 784 antimicrobial test results,
76 for Gram-positives and 708 for Gram-negatives. Five (42\%) of the Gram-positive and 42 (79\%) of the Gramnegative isolates were resistant to one or more antimicrobials. Interestingly, among Klebsiella pneumoniae strains, 10 (48\%) were carbapenemase-producers, 9 of these codified for $b l a_{\mathrm{KPC}}$ and one for $b l a_{\mathrm{VIM}}$ genes, identified by the off-label use of the Xpert ${ }^{\circledR}$ Carba-R test (Cepheid, Sunnyvale, CA, United States) [28]. Four KPC-Klebsiella pneumoniae isolates were resistant to colistin, too. Six E. coli strains (32\%) were resistant to one or more third-generation cephalosporins, and one was resistant to colistin. Among Gram-positives, one E. faecium isolate (33\%) codified for vanA gene and one S. aureus (25\%) was a methicillin-resistant strain.

3.3. Polymicrobial Blood Cultures. Among 67 BCs, 7 (10\%) appeared to be polymicrobial when subcultured on blood agar plates. Most likely the proportion of the second microorganism was very low, since direct identification by MALDI-TOF MS detected only the first one, with a high score (Table 3). Pheno failed the ID and the AST for four polymicrobial BCs (Enterococcus/Pseudomonas; Enterococcus/Streptococcus; Enterobacter/Staphylococcus; Klebsiella/ Bacillus); for the couple Klebsiella/Staphylococcus it identified and provided the AST only for the Gram-negative, for the couple Klebsiella/Enterococcus it identified both the microorganisms and provided the AST for the Enterococcus spp.; finally, for the couple Klebsiella/Citrobacter it identified only Klebsiella spp. without providing the AST.

3.4. Identification of BSI Pathogens. Correct rapid identification by MALDI-TOF MS was achieved in all the monomicrobial BCs, although for two Enterobacter cloacae and one S. aureus infections the identification score was low (Table 2). 


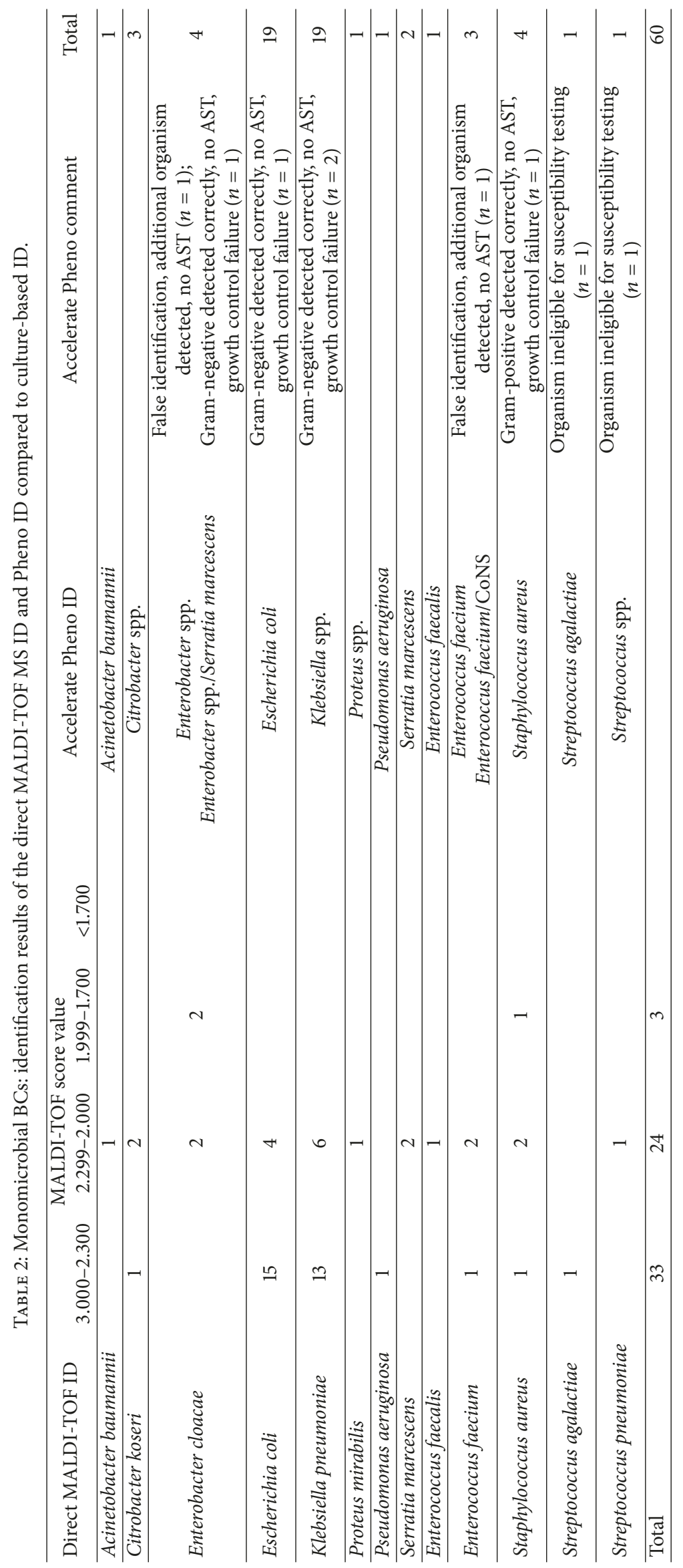




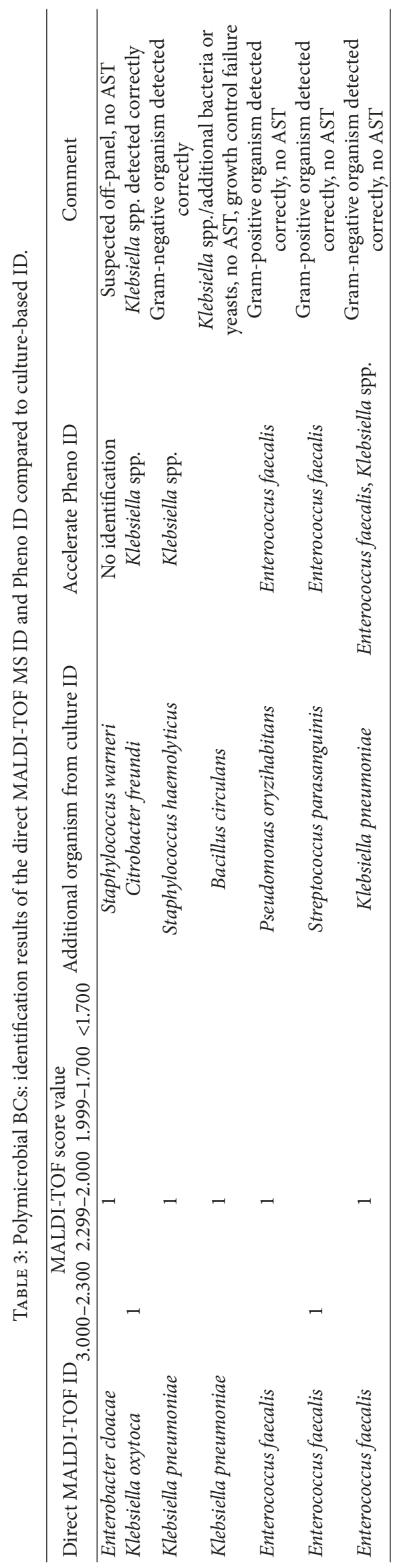


TABLE 4: Antimicrobial susceptibility testing results of Pheno system compared to the routine SensiTitre system. The summary of discrepancies in percent is reported in brackets. ${ }^{*}$ Cefotaxime result was predicted according to ceftriaxone SIR classification (EUCAST rules v1.2.0.4).

(a)

\begin{tabular}{|c|c|c|c|c|c|}
\hline Antimicrobial agent & $\begin{array}{l}\text { Number of } \\
\text { very major } \\
\text { errors (\%) }\end{array}$ & $\begin{array}{c}\text { Number of } \\
\text { major errors } \\
(\%)\end{array}$ & $\begin{array}{c}\text { Number of } \\
\text { minor errors } \\
(\%)\end{array}$ & AST agreement (\%) & Total \\
\hline \multicolumn{6}{|l|}{ Gram-positives } \\
\hline Ampicillin & $0 / 2$ & $1 / 2(50.0)$ & $0 / 4$ & $3 / 4(75.0)$ & 4 \\
\hline Ceftaroline & $0 / 0$ & $0 / 3$ & $0 / 3$ & $3 / 3(100.0)$ & 3 \\
\hline Doxycycline & $0 / 0$ & $0 / 2$ & $0 / 2$ & $2 / 2(100.0)$ & 2 \\
\hline Erythromycin & $0 / 1$ & $0 / 2$ & $0 / 3$ & $3 / 3(100.0)$ & 3 \\
\hline Trimethoprim-sulfamethoxazole & $0 / 0$ & $0 / 3$ & $0 / 3$ & $3 / 3(100.0)$ & 3 \\
\hline Daptomycin & $0 / 0$ & $0 / 5$ & $0 / 5$ & $5 / 5(100.0)$ & 5 \\
\hline Linezolid & $0 / 0$ & $0 / 7$ & $0 / 7$ & $7 / 7(100.0)$ & 7 \\
\hline Vancomycin & $0 / 1$ & $1 / 6(16.7)$ & $0 / 7$ & $6 / 7(85.7)$ & 7 \\
\hline Cefoxitin & $0 / 0$ & $0 / 2$ & $0 / 2$ & $2 / 2(100.0)$ & 2 \\
\hline MLS screening & $0 / 0$ & $0 / 2$ & $0 / 2$ & $2 / 2(100.0)$ & 2 \\
\hline Total & $0 / 4$ & $2 / 34(5.9)$ & $0 / 38$ & $36 / 38(94.7)$ & 38 \\
\hline
\end{tabular}

(b)

\begin{tabular}{|c|c|c|c|c|c|}
\hline Antimicrobial agent & $\begin{array}{l}\text { Number of } \\
\text { very major } \\
\text { errors (\%) }\end{array}$ & $\begin{array}{l}\text { Number of } \\
\text { major errors } \\
(\%)\end{array}$ & $\begin{array}{l}\text { Number of } \\
\text { minor errors } \\
(\%)\end{array}$ & AST agreement (\%) & Total \\
\hline \multicolumn{6}{|l|}{ Gram-negatives } \\
\hline Ampicillin/sulbactam & $0 / 27$ & $3 / 7(42.9)$ & $0 / 34$ & $31 / 34(91.2)$ & 34 \\
\hline Piperacillin/tazobactam & 2/15 (13.3) & $3 / 28(10.7)$ & $4 / 46(8.7)$ & $37 / 46(80.4)$ & 46 \\
\hline Cefazoline & $0 / 1$ & $0 / 0$ & $0 / 1$ & 1/1 (100.0) & 1 \\
\hline Cefepime & $0 / 13$ & $1 / 30(3.3)$ & $6 / 46(13.0)$ & $39 / 46(84.8)$ & 46 \\
\hline Ceftazidime & $0 / 15$ & $1 / 28(3.6)$ & $2 / 45(4.4)$ & $42 / 45(93.3)$ & 45 \\
\hline Cefotaxime* & $0 / 0$ & $0 / 1$ & $0 / 1$ & $1 / 1(100.0)$ & 1 \\
\hline Ertapenem & 1/9 (11.1) & $0 / 35$ & $1 / 44(2.3)$ & $42 / 44(95.5)$ & 44 \\
\hline Meropenem & $1 / 9(11.1)$ & $0 / 37$ & $5 / 46(10.9)$ & $40 / 46(87.0)$ & 46 \\
\hline Gentamicin & $0 / 7$ & $3 / 38(7.9)$ & $1 / 45(2.2)$ & $41 / 45(91.1)$ & 45 \\
\hline Amikacin & $0 / 9$ & $0 / 33$ & $5 / 46(10.9)$ & $41 / 46(89.1)$ & 46 \\
\hline Tobramycin & $0 / 0$ & $0 / 2$ & $0 / 2$ & $2 / 2(100.0)$ & 2 \\
\hline Ciprofloxacin & $0 / 22$ & $0 / 22$ & $2 / 46(4.4)$ & $44 / 46(95.7)$ & 46 \\
\hline Colistin & $0 / 5$ & $2 / 38(5.3)$ & $0 / 43$ & $41 / 43(95.4)$ & 43 \\
\hline Total & $4 / 132(3.0)$ & $13 / 299(4.4)$ & $26 / 445(5.8)$ & $402 / 445(90.3)$ & 445 \\
\hline
\end{tabular}

To assess the reproducibility of the identification, the bacteria were spotted in duplicate and the results revealed $100 \%$ concordance and similar scores. Pheno was not able to provide results for 7 monomicrobial cultures, for 3 of these probably because the time elapsed from positivity to the $\mathrm{BC}$ processing was close to 16 hours, thus outside company recommendations. For 4 BCs (7\%), the control growth failed without other explanations. These results show that Pheno processed $88 \%$ of BCs introduced in the instrument. Furthermore, Pheno identified an additional microorganism in 2 infections classified as monomicrobial by routine culture method. Particularly, a strain of Serratia marcescens was identified in a BC positive on plate as Enterobacter cloacae and a CoNS in a BC positive on plate as Enterococcus faecium. Therefore, the proportion of corrected identified microorganisms in monomicrobial BCs was $97 \%$.

3.5. Antimicrobial Susceptibility for Pheno. Antimicrobial susceptibility results for Pheno were produced for 52 isolates with a total of 483 antimicrobial test results (38 Grampositives and 445 Gram-negatives). Table 4(a) lists the summary of Pheno discrepancy testing results for Grampositives. Among them, 36 out of the 38 tests performed were concordant with the routine SensiTitre; 2 MEs (5.9\%) were found for ampicillin and vancomycin, with a CA of $94.7 \%$ and EA of $82.9 \%$. For a single S. aureus isolate, Pheno failed 
the MIC calculation of the cefoxitin screening test and did not provide the result. The AST results of Gram-negatives, the total percent errors as well as the percent error for each antimicrobial agent are shown in Table 4(b). Among Gramnegatives, 4 VMEs (3.0\%) involved piperacillin/tazobactam $(n=2)$, ertapenem $(n=1)$, and meropenem $(n=1)$. For 3 carbapenemase-producing Klebsiella pneumoniae isolates, the meropenem MIC value was below the breakpoint; nevertheless, we considered the result as concordant, since Pheno provided a note advising for the presence of carbapenemaseproducer microorganism. Thirteen MEs (4.6\%) were referred to ampicillin/sulbactam $(n=3)$, piperacillin/tazobactam $(n=3)$, gentamicin $(n=3)$, colistin $(n=2)$, cefepime $(n=1)$, and ceftazidime $(n=1)$. There were $26 \mathrm{MiEs}$ $(5.84 \%)$ for cefepime $(n=6)$, meropenem $(n=5)$, amikacin $(n=5)$, piperacillin/tazobactam $(n=4)$, ceftazidime $(n=2)$, ciprofloxacin $(n=2)$, ertapenem $(n=1)$, and gentamicin $(n=1)$. Since with Alfred 60AST polymicrobial cultures could not be processed, we decided to remove them from Pheno analysis also. Excluding the polymicrobial BCs, 428 tests for Gram-negatives and 34 tests for Gram-positives were analyzed. The CA and EA of Gram-positives were $100 \%$ and $87 \%$, respectively, while for Gram-negatives the CA was $90.6 \%$ (including 4 VMEs (3.2\%), 13 MEs (4.5\%), and 23 MiEs $(4.4 \%)$ ) and the EA was $81.4 \%$. These results show that polymicrobial cultures slightly affect the CA for Pheno, mostly for Gram-positive bacteria. Further studies, mainly focalized on polymicrobial BCs, might be necessary in order to assess the performances of Pheno on this type of samples, as also reported elsewhere [29]. The overall categorical agreement of the 496 AST results was $91.3 \%$ with minor, major, and very major errors occurring at a rate of 5.0, 4.1 , and $3.1 \%$, respectively.

3.6. Antimicrobial Susceptibility for Alfred 60AST. Antimicrobial susceptibility results for Alfred 60AST were produced for 58 isolates with a total of 405 antimicrobial test results (47 Gram-positives and 358 Gram-negatives). The time needed to reach a bacterial density corresponding to $0.5 \mathrm{McFar}-$ land ranged between 1-2 hours for Gram-negatives (median time $1 \mathrm{~h}$ and $40 \mathrm{~min}, \pm 18 \mathrm{~min}$ ) and $1-3$ hours for Grampositives (median time $1 \mathrm{~h}$ and $55 \mathrm{~min}, \pm 39 \mathrm{~min}$ ), depending on bacterial species. For Gram-positive bacteria, the CA was 85.1\%. Seven MEs (17.1\%) were found for linezolid ( $n=$ $1)$, vancomycin $(n=2)$, and teicoplanin $(n=4)$. For glycopeptides, we experienced several tests with MEs [27, 30]; however, with a careful observation of growth curves, it was possible to realize that the interpretation was incorrect (Figure 2). Hence, with a manual correction of ambiguous growth curves, the agreement for Gram-positives was of $95.7 \%$, with 2 MEs only (4.9\%). Table 5(a) lists the summary of Alfred 60AST discrepancy testing results for Gram-positives. As reported in Table 5(b) the CA for Gram-negatives was 91.1\%. Sixteen VMEs (15.4\%) involved levofloxacin $(n=5)$, gentamicin $(n=4)$, colistin $(n=2)$, ceftazidime $(n=$ $2)$, cefotaxime $(n=1)$, and trimethoprim-sulfamethoxazole $(n=2)$. There were 11 MEs $(4.4 \%)$ involving trimethoprimsulfamethoxazole $(n=1)$, gentamicin $(n=1)$, meropenem $(n=2)$, and piperacillin/tazobactam $(n=7)$. Finally, 5
MiEs $(1.4 \%)$ were detected among ceftazidime $(n=2)$ and piperacillin/tazobactam $(n=3)$. The overall CA of the 405 AST results was of $91.6 \%$ with minor, major, and very major errors occurring at a rate of $1.2,4.5$, and $14.5 \%$, respectively.

3.7. Direct Comparison of Alfred 60AST versus Pheno. Direct comparison between the two methodologies was possible for 261 AST results. Two hundred and twenty-five tests (86.2\%) were concordant: among these, $85.4 \%$ for Gram-negatives and $95.2 \%$ for Gram-positives. Five concordant tests (1.9\%) were discordant with SensiTitre, particularly gentamicin $(n=$ 1), ceftazidime $(n=1)$, and piperacillin/tazobactam ( $n=$ 3). In the latter cases, we used the MIC test strip and the SensiTitre results were confirmed. These results suggest that the majority of issues for both methods were represented by piperacillin/tazobactam, which is known to be unstable [31]. Details of AST results for each antibiotic are presented in Table 6.

\section{Conclusions}

Regardless of its precise definition, sepsis is recorded as the most expensive condition in hospitals all over the world [1, $3,32]$. Early appropriate antimicrobial regimen, especially in the case of multidrug resistant bacteria, is pivotal to decrease mortality and may contribute to reducing healthcare costs $[33,34]$. An additional predictable benefit is a reduction in the escalating rate of resistances, estimated to cause 10 million deaths per year worldwide in the next 30 years, with a cumulative economic cost of US\$100 trillion [32]. The optimal duration of therapy for a bloodstream infection would be long enough to effectively eradicate infection and prevent relapse, while short enough to limit adverse effects, avoid secondary infections by opportunistic pathogens, and minimize selective pressure for antibiotic resistance [4]. In our hospital, given the high prevalence of multidrug resistant pathogens, there is an urgent need of rapid antimicrobial susceptibility test results during sepsis. For this reason, we evaluated the performance of the Pheno system and MALDI-TOF MS coupled with Alfred 60AST system for rapid identification and antimicrobial susceptibility tests of microorganisms directly from positive blood cultures. To our knowledge, this is the first evaluation of the two methodologies in comparison to the SensiTitre broth microdilution assay, both on Gram-positive and Gram-negative bacteria.

The Pheno system performed well for the identification of bloodstream pathogens, correctly identifying $97 \%$ of microorganisms. These results are in agreement with a recent study conducted only on Gram-negative bacteria [29]. It is worthy of notice that the system failed the growth control for various reasons for $12 \%$ monomicrobial BCs and, since Pheno is a closed system, there is no possibility for the operator to intervene and fix the eventual inconvenience. The failed tests are slightly lower than the proportion reported elsewhere [35]; nevertheless, the studies about this aspect are still partial. Pheno presents the limitation of processing only fresh BCs (within 8 hours after a BC turns positive). Furthermore, for many microorganisms, only the genus was available; however, identification to the species level may be 
TABLE 5: Antimicrobial susceptibility testing results of Alfred 60AST system compared to the routine SensiTitre system. Data are numbers (with percentages) of bacterial isolates for which the AST was performed. The summary of discrepancies as well as the AST agreement is reported in brackets.

(a)

\begin{tabular}{|c|c|c|c|c|c|}
\hline Antimicrobial agent & $\begin{array}{l}\text { Number of } \\
\text { very major } \\
\text { errors (\%) }\end{array}$ & $\begin{array}{c}\text { Number of } \\
\text { major errors } \\
(\%)\end{array}$ & $\begin{array}{l}\text { Number of } \\
\text { minor errors } \\
(\%)\end{array}$ & $\begin{array}{c}\text { AST } \\
\text { agreement } \\
(\%)\end{array}$ & Total \\
\hline \multicolumn{6}{|l|}{ Gram-positives } \\
\hline Ampicillin & $0 / 3$ & $0 / 2$ & $0 / 5$ & $5 / 5(100.0)$ & 5 \\
\hline Daptomycin & $0 / 0$ & $0 / 8$ & $0 / 8$ & $8 / 8(100.0)$ & 8 \\
\hline Linezolid & $0 / 0$ & $1 / 10(10.0)$ & $0 / 10$ & 9/10 (90.0) & 10 \\
\hline Vancomycin & $0 / 1$ & $2 / 6(33.3)$ & $0 / 7$ & $5 / 7(71.4)$ & 7 \\
\hline Teicoplanin & $0 / 1$ & $4 / 8(50.0)$ & $0 / 9$ & $5 / 9(55.6)$ & 9 \\
\hline Cefoxitin & $0 / 1$ & $0 / 3$ & $0 / 4$ & $4 / 4(100.0)$ & 4 \\
\hline Clindamycin & $0 / 0$ & $0 / 4$ & $0 / 4$ & $4 / 4(100.0)$ & 4 \\
\hline Total & $0 / 6$ & $7 / 41(17.1)$ & $0 / 47$ & $40 / 47(85.1)$ & 47 \\
\hline
\end{tabular}

(b)

\begin{tabular}{|c|c|c|c|c|c|}
\hline Antimicrobial agent & $\begin{array}{l}\text { Number of } \\
\text { very major } \\
\text { errors (\%) }\end{array}$ & $\begin{array}{c}\text { Number of } \\
\text { major errors } \\
(\%)\end{array}$ & $\begin{array}{c}\text { Number of } \\
\text { minor errors } \\
(\%)\end{array}$ & $\begin{array}{c}\text { AST } \\
\text { agreement } \\
(\%)\end{array}$ & Total \\
\hline \multicolumn{6}{|l|}{ Gram-negatives } \\
\hline Piperacillin/tazobactam & $0 / 15$ & $7 / 26(26.9)$ & $3 / 44(6.8)$ & $34 / 44(77.3)$ & 44 \\
\hline Ceftazidime & $2 / 14(14.3)$ & $0 / 30$ & $2 / 46(4.4)$ & $42 / 46(91.3)$ & 46 \\
\hline Cefotaxime & $1 / 14(7.1)$ & $0 / 31$ & $0 / 45$ & $44 / 45(97.8)$ & 45 \\
\hline Meropenem & $0 / 8$ & $2 / 38(5.3)$ & $0 / 46$ & $44 / 46(95.7)$ & 46 \\
\hline Gentamicin & $4 / 8(50.0)$ & $1 / 37(2.7)$ & $0 / 45$ & $40 / 45(88.9)$ & 45 \\
\hline Colistin & $2 / 6(33.3)$ & $0 / 37$ & $0 / 43$ & $41 / 43(95.4)$ & 43 \\
\hline Levofloxacin & $5 / 20(25.0)$ & $0 / 24$ & $0 / 44$ & $39 / 44(88.6)$ & 44 \\
\hline Trimethoprim-sulfamethoxazole & 2/18 (11.1) & $1 / 23(4.4)$ & $0 / 41$ & $38 / 41(92.7)$ & 41 \\
\hline Cefuroxime & $0 / 1$ & $0 / 3$ & $0 / 4$ & $4 / 4(100.0)$ & \\
\hline Total & $16 / 104(15.4)$ & $11 / 249(4.4)$ & $5 / 358(1.4)$ & $326 / 358(91.1)$ & 358 \\
\hline
\end{tabular}
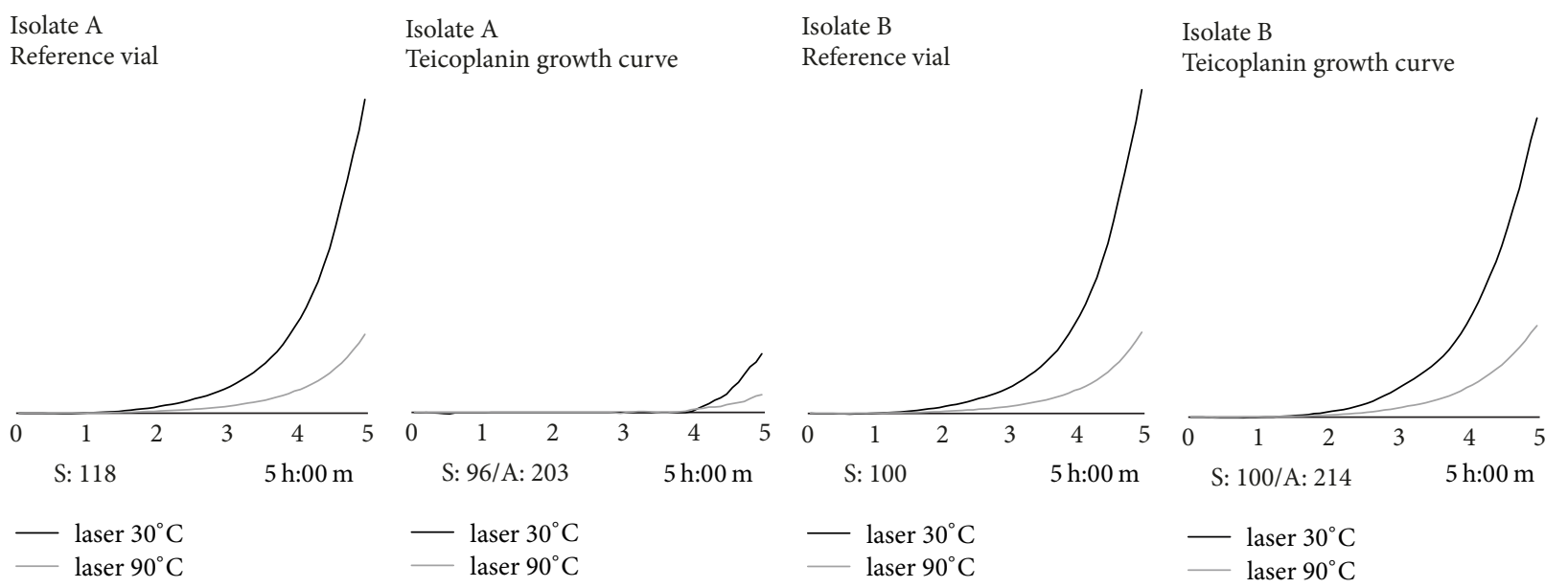

FIGURE 2: Isolate A represents an example of a false teicoplanin-resistant strain: in the presence of the drug, the microorganism seems to start growing in the last 30 minutes of the analysis. Isolate B represents a true teicoplanin-resistant strain: the growth curve in the presence of the drug is comparable to the reference vial. The horizontal bar under the figures represents incubation time ( 5 hours). Laser $30^{\circ} \mathrm{C}$ and laser $90^{\circ} \mathrm{C}$ represent the two detectors of the instrument. S sample; A antibiotics. 
TABLE 6: AST results of Alfred 60AST compared to Pheno. The summary of discrepancies and AST agreement in percent is reported in brackets (\%).

(a)

\begin{tabular}{lccc}
\hline Antimicrobial agent & Number of discrepancies (\%) & AST agreement (\%) & Total \\
\hline Gram-positives & & & \\
Ampicillin & $0 / 3$ & $3 / 3(100.0)$ & 3 \\
Daptomycin & $0 / 4$ & $6 / 4(100.0)$ & 4 \\
Linezolid & $0 / 6$ & $4 / 5(80.0)$ & 6 \\
Vancomycin & $1 / 5(20.0)$ & $2 / 2(100.0)$ & 5 \\
Cefoxitin & $0 / 2$ & $1 / 1(100.0)$ & 2 \\
Clindamycin & $0 / 1$ & $20 / 21(95.2)$ & 1 \\
Total & $1 / 21(4.8)$ & & 21 \\
\hline
\end{tabular}

(b)

\begin{tabular}{lccc}
\hline Antimicrobial agent & Number of discrepancies (\%) & AST agreement (\%) & Total \\
\hline Gram-negatives & & & \\
Piperacillin/tazobactam & $11 / 40(27.5)$ & $29 / 40(72.5)$ & $37 / 41(90.2)$ \\
Ceftazidime & $4 / 41(9.8)$ & $36 / 39(92.3)$ & 40 \\
Ceftriaxone & $3 / 39(7.7)$ & $1 / 1(100.0)$ & 39 \\
Cefotaxime & $0 / 1$ & $34 / 41(82.9)$ & 1 \\
Meropenem & $7 / 41(17.1)$ & $34 / 40(85.0)$ & 41 \\
Gentamicin & $6 / 40(15.0)$ & $34 / 38(89.5)$ & 40 \\
Colistin & $4 / 38(10.5)$ & $205 / 240(85.4)$ & 240 \\
Total & $35 / 240(14.6)$ & & \\
\hline
\end{tabular}

important for infection control purposes and epidemiological studies. In addition, Pheno detected a second organism in 2 monomicrobial BCs according to the routine culture method, precisely Serratia marcescens in a BC positive only for Enterobacter spp., and CoNS coupled with an Enterococcus faecium strain. Performance on AST data was good both for Grampositive and Gram-negative microorganisms, with an overall categorical agreement of $100 \%$ and $90.6 \%$, respectively. There were 4 false-susceptible and 13 false-resistant results, affecting mainly $\beta$-lactams, gentamicin and colistin. The categorical and essential agreements obtained in the present study were slightly lower (CA 91.3\% versus $95.5 \%$ and EA $81.8 \%$ versus 95.1\%) than data available in a recently published article by Charnot-Katsikas and colleagues [35]. A possible explanation is that they used Vitek2 (bioMérieux) as a comparator, a system which operates on fewer dilutions and so may be less precise than the reference method used in the present study. A key point of Pheno is that the instrument is user-friendly and does not require skillful technicians or a well-trained staff. In addition, the time required to obtain a complete report including both the ID and AST was rapid: 3-5 min for cartridge set up, 80-120 min for ID, and about 5-6 hours for AST.

The Alfred 60AST system was coupled with MALDITOF MS for direct identification and AST in positive blood cultures. MALDI-TOF MS performances were optimal, since a correct identification was achieved in all the monomicrobial BCs analyzed. An interesting meta-analysis about direct MALDI-TOF ID from positive blood cultures suggested that MALDI-TOF provides highly accurate identification of Gram-negative bacteria at the species level, while for Gram-positive bacteria overall accuracy is moderate [36]. On the contrary, we obtained optimal results also with Grampositives, probably because a valid protein extraction protocol was used. Regarding AST, Alfred 60AST performances were good, with an overall categorical agreement of $91.0 \%$ and $95.7 \%$ for Gram-negative and Gram-positive bacteria, respectively. The majority of problems involved the glycopeptides, particularly teicoplanin (Alifax). According to some experiments performed in our laboratory, glycopeptides (Alifax) lose their effectiveness when close to the expiration date, probably because the drug powder deteriorated over time. For this reason, the cap on the vial containing the lyophilized antibiotic was replaced by the company with a new version capable of guaranteeing greater resistance to external agents (e.g., humidity in the refrigerator cells), preserving the effectiveness of the drug. There were 16 false-susceptible and 18 false-resistant results, affecting mainly $\beta$-lactams, gentamicin, levofloxacin, and glycopeptides. Further, 2 VMEs affecting colistin were due to the duration of the test. Apparently, 3 hours were not enough to allow the resistance to be revealed by the algorithm; thus, incubation time for the colistin test was extended up to 5 hours. One of the main aspects of Alfred 60AST is the plasticity of the system, since there is the possibility of eventually intervening in the course of the AST. On the other hand, a trained staff, able to interpret the results and particularly the microbial growth curves, is required. Notably, the microbiology laboratory can decide the antibiotic to insert in the AST panel, according to patient needs. The time required to obtain a complete result including 
both the ID and AST was about 6 hours: 3-5 min for setting up vials, 15-20 min for MALDI-TOF MS ID, and about 4-6 hours for AST, depending on the chosen antibiotics.

In conclusion, both methodologies provided comparable results, showing no statistically significant differences. AST preliminary results were reliable for both and useful to start a proper antibiotic treatment, which can be confirmed afterwards with the traditional AST. The time to obtain ID and AST as well as costs are lower for Alfred 60AST combined with MALDI-TOF MS. On the other hand, Pheno provides both identification and MIC determination in one cartridge. Although certainly more expensive, Pheno can be useful in medium and small laboratories and when MIC values are necessary for an appropriate therapy. Both systems allow us to establish a proper diagnostic stewardship in order to hinder sepsis and minimize the spread of bacterial resistance.

\section{Conflicts of Interest}

The authors declare no conflicts of interest related to this study.

\section{References}

[1] M. Singer, C. S. Deutschman, C. W. Seymour et al., "The third international consensus definitions for sepsis and septic shock (sepsis-3)," The Journal of the American Medical Association, vol. 315, no. 8, pp. 801-810, 2016.

[2] M. J. Hall, S. N. Williams, C. J. DeFrances, and A. Golosinskiy, "Inpatient care for septicemia or sepsis: a challenge for patients and hospitals.," NCHS Data Brief, no. 62, pp. 1-8, 2011.

[3] H. Arefian, S. Heublein, A. Scherag et al., "Hospital-related cost of sepsis: A systematic review," Infection, vol. 74, no. 2, pp. 107117, 2017.

[4] T. C. Havey, R. A. Fowler, R. Pinto, M. Elligsen, and N. Daneman, "Duration of antibiotic therapy for critically ill patients with bloodstream infections: A retrospective cohort study," Canadian Journal of Infectious Diseases \& Medical Microbiology, vol. 24, no. 3, pp. 129-137, 2013.

[5] US Department of Health and Human Services, Antibiotic/Antimicrobial Resistance, Center for Disease Control and Prevention, 2013, https://www.cdc.gov/drugresistance/threat-report2013/pdf/ar-threats-2013-508.pdf.

[6] B. Spellberg, M. Blaser, R. J. Guidos et al., "Combating antimicrobial resistance: policy recommendations to save lives," Clinical Infectious Diseases, vol. 52, no. 5, pp. S397-S428, 2011.

[7] J. L. Liscio, M. V. Mahoney, and E. B. Hirsch, "Ceftolozane/tazobactam and ceftazidime/avibactam: two novel $\beta$-lactam/ $\beta$-lactamase inhibitor combination agents for the treatment of resistant Gram-negative bacterial infections," International Journal of Antimicrobial Agents, vol. 46, no. 3, pp. 266-271, 2015.

[8] R. Sharma, D. Francois, and M. R. Hammerschlag, "New antimicrobial agents for the treatment of staphylococcal infections in children," Pediatric Clinics of North America, vol. 64, no. 6, pp. 1369-1387, 2017.

[9] A. Kothari, M. Morgan, and D. A. Haake, "Emerging technologies for rapid identification of bloodstream pathogens," Clinical Infectious Diseases, vol. 59, no. 2, pp. 272-278, 2014.
[10] J. H. Jorgensen and M. J. Ferraro, "Antimicrobial susceptibility testing: a review of general principles and contemporary practices," Clinical Infectious Diseases, vol. 49, no. 11, pp. 1749-1755, 2009.

[11] P. Dalgaard, T. Ross, L. Kamperman, K. Neumeyer, and T. A. McMeekin, "Estimation of bacterial growth rates from turbidimetric and viable count data," International Journal of Food Microbiology, vol. 23, no. 3-4, pp. 391-404, 1994.

[12] J. Q. Boedicker, L. Li, T. R. Kline, and R. F. Ismagilov, "Detecting bacteria and determining their susceptibility to antibiotics by stochastic confinement in nanoliter droplets using plug-based microfluidics," Lab on a Chip, vol. 8, no. 8, pp. 1265-1272, 2008.

[13] P. Kinnunen, B. H. McNaughton, T. Albertson et al., "Selfassembled magnetic bead biosensor for measuring bacterial growth and antimicrobial susceptibility testing," Small, vol. 8, no. 16, pp. 2477-2482, 2012.

[14] M. Kalashnikov, J. C. Lee, J. Campbell, A. Sharon, and A. F. Sauer-Budge, "A microfluidic platform for rapid, stress-induced antibiotic susceptibility testing of Staphylococcus aureus," Lab on a Chip, vol. 12, no. 21, pp. 4523-4532, 2012.

[15] N. G. Schoepp, T. S. Schlappi, M. S. Curtis et al., "Rapid pathogen-specific phenotypic antibiotic susceptibility testing using digital LAMP quantification in clinical samples," Science Translational Medicine, vol. 9, no. 410, Article ID aal3693, 2017.

[16] C. Mauri, L. Principe, S. Bracco et al., "Identification by mass spectrometry and automated susceptibility testing from positive bottles: A simple, rapid, and standardized approach to reduce the turnaround time in the management of blood cultures," BMC Infectious Diseases, vol. 17, no. 1, article no. 749, 2017.

[17] W. Florio, P. Morici, E. Ghelardi, S. Barnini, and A. Lupetti, "Recent advances in the microbiological diagnosis of bloodstream infections," Critical Reviews in Microbiology, vol. 44, no. 3, pp. 351-370, 2018.

[18] C. Fontana, M. Favaro, S. Minelli, M. C. Bossa, A. Altieri, and C. Favalli, "A novel culturing system for fluid samples," Medical Science Monitor, vol. 15, no. 2, pp. BR55-BR60, 2009.

[19] C. Fontana, M. Favaro, M. C. Bossa et al., "Improved diagnosis of central venous catheter-related bloodstream infections using the HB\&L UROQUATTRO ${ }^{\mathrm{TM}}$ system," European Journal of Clinical Microbiology \& Infectious Diseases, vol. 31, no. 11, pp. 3139-3144, 2012.

[20] S. Lahanas, G. Stathopoulos, R. C. Chan, and S. J. Van Hal, "Evaluation of the Alfred 60/AST device as a screening test for urinary tract infections," Journal of Clinical Microbiology, vol. 51, no. 10, pp. 3406-3408, 2013.

[21] S. Barnini, V. Brucculeri, P. Morici, E. Ghelardi, W. Florio, and A. Lupetti, "A new rapid method for direct antimicrobial susceptibility testing of bacteria from positive blood cultures," BMC Microbiology, vol. 16, article 185, 2016.

[22] S. Barnini, E. Ghelardi, V. Brucculeri, P. Morici, and A. Lupetti, "Rapid and reliable identification of Gram-negative bacteria and Gram-positive cocci by deposition of bacteria harvested from blood cultures onto the MALDI-TOF plate Clinical microbiology and vaccines," BMC Microbiology, vol. 15, article 124, 2015.

[23] A. R. Marra, M. B. Edmond, B. A. Forbes, R. P. Wenzel, and G. M. L. Bearman, "Time to blood culture positivity as a predictor of clinical outcome of Staphylococcus aureus bloodstream infection," Journal of Clinical Microbiology, vol. 44, no. 4, pp. 1342-1346, 2006.

[24] X. García, C. Sabatier, R. Ferrer et al., "Differential time to positivity of blood cultures: A valid method for diagnosing 
catheter-related bloodstream infections in the intensive care unit," Medicina Intensiva, vol. 36, no. 3, pp. 169-176, 2012.

[25] Y. Ning, R. Hu, G. Yao, and S. Bo, "Time to positivity of blood culture and its prognostic value in bloodstream infection," European Journal of Clinical Microbiology \& Infectious Diseases, vol. 35, no. 4, pp. 619-624, 2016.

[26] V. Brucculeri, O. Paolilli, A. M. Zucal, A. Lupetti, and S. Barnini, "Il tempo di positività dell'emocoltura," in Proceedings of the XLIV AMCLI, Associazione Microbiologi Clinici Italiani, 2015, poster no. P003.

[27] C. Giordano, V. Brucculeri, C. Ceppatelli, L. Lelli, and S. Barnini, "A new and fast laboratory workflow for the diagnosis and care of sepsis," in Proceedings of the 27th ECCMID, European Congress of Clinical Microbiology and Infectious diseases, 2017, poster no. P2067.

[28] C. Giordano, V. Brucculeri, L. Lelli, and S. Barnini, "Ridurre KPC con ogni metodo: l'esperienza di Pisa," in Proceedings of the XLV AMCLI, Associazione Microbiologi Clinici Italiani, 2016, poster no. P106.

[29] M. Marschal, J. Bachmaier, I. Autenrieth, P. Oberhettinger, M. Willmann, and S. Petera, "Evaluation of the accelerate pheno system for fast identification and antimicrobial susceptibility testing from positive blood cultures in bloodstream infections caused by gram-negative pathogens," Journal of Clinical Microbiology, vol. 55, no. 7, pp. 2116-2126, 2017.

[30] C. Giordano, V. Brucculeri, L. Lelli, A. Lupetti, and S. Barnini, "Improving the concordance between the rapid antimicrobial susceptibility test and the traditional semi-automated antimicrobial susceptibility test," in Proceedings of the 44。 SIM, Società Italiana di Microbiologia, 2016, poster no. P63.

[31] M. Mathew, V. D. Gupta, and C. Bethea, "Stability of piperacillin sodium in the presence of tazobactam sodium in 5\% dextrose and normal saline injections," Journal of Clinical Pharmacy and Therapeutics, vol. 19, no. 6, pp. 397-399, 1994.

[32] J. O’Neill, “Tackling drug-resistant infections globally: final report and recommendations," in Review on Antimicrobial Resistance, 2016, https://amr-review.org/sites/default/files/160518_ Final\%20paper_with\%20cover.pdf.

[33] J. Barenfanger, C. Drake, and G. Kacich, "Clinical and financial benefits of rapid bacterial identification and antimicrobial susceptibility testing," Journal of Clinical Microbiology, vol. 37, no. 5, pp. 1415-1418, 1999.

[34] J. Barenfanger, D. R. Graham, L. Kolluri et al., "Decreased mortality associated with prompt gram staining of blood cultures," American Journal of Clinical Pathology, vol. 130, no. 6, pp. 870876, 2008.

[35] A. Charnot-Katsikas, V. Tesic, N. Love et al., "Use of the Accelerate Pheno System for Identification and Antimicrobial Susceptibility Testing of Pathogens in Positive Blood Cultures and Impact on Time to Results and Workflow," Journal of Clinical Microbiology, vol. 56, no. 1, p. e01166-17, 2018.

[36] J. Ruiz-Aragón, M. Ballestero-Téllez, B. Gutiérrez-Gutiérrez, M. de Cueto, J. Rodríguez-Baño, and Á. Pascual, "Direct bacterial identification from positive blood cultures using matrixassisted laser desorption/ionization time-of-flight (MALDITOF) mass spectrometry: A systematic review and meta-analysis," Enfermedades Infecciosas y Microbiología Clínica, 2017, In Press. 


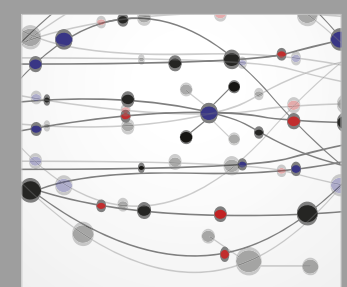

The Scientific World Journal
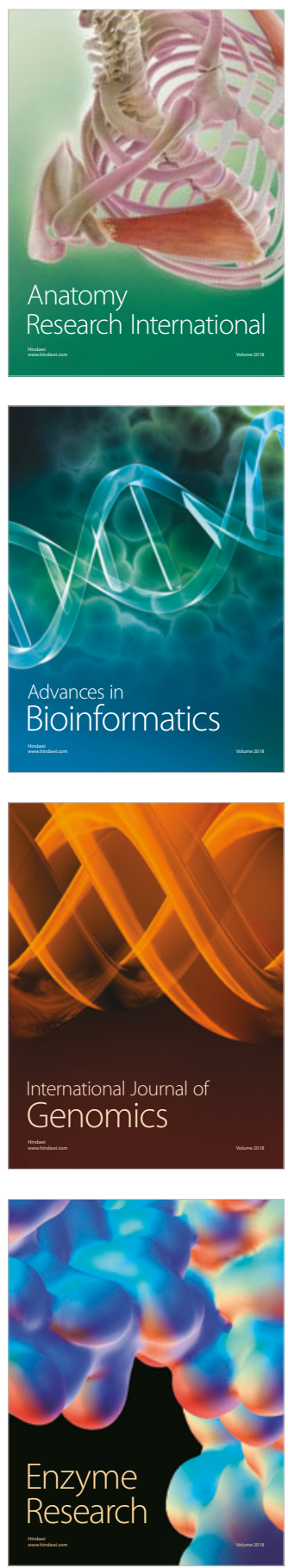
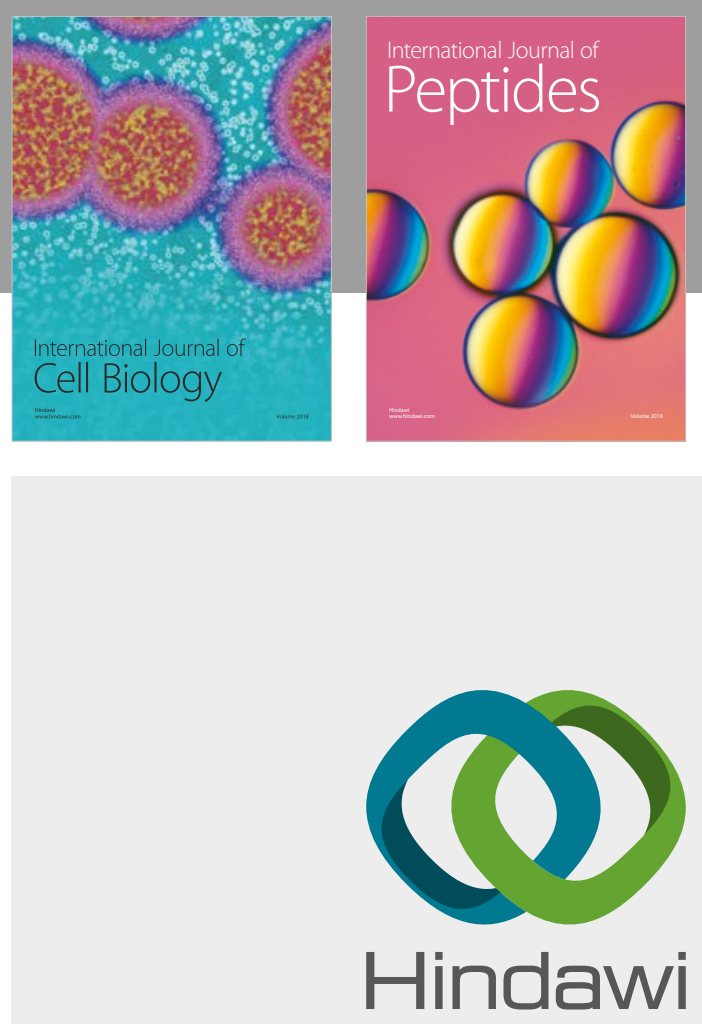

Submit your manuscripts at

www.hindawi.com
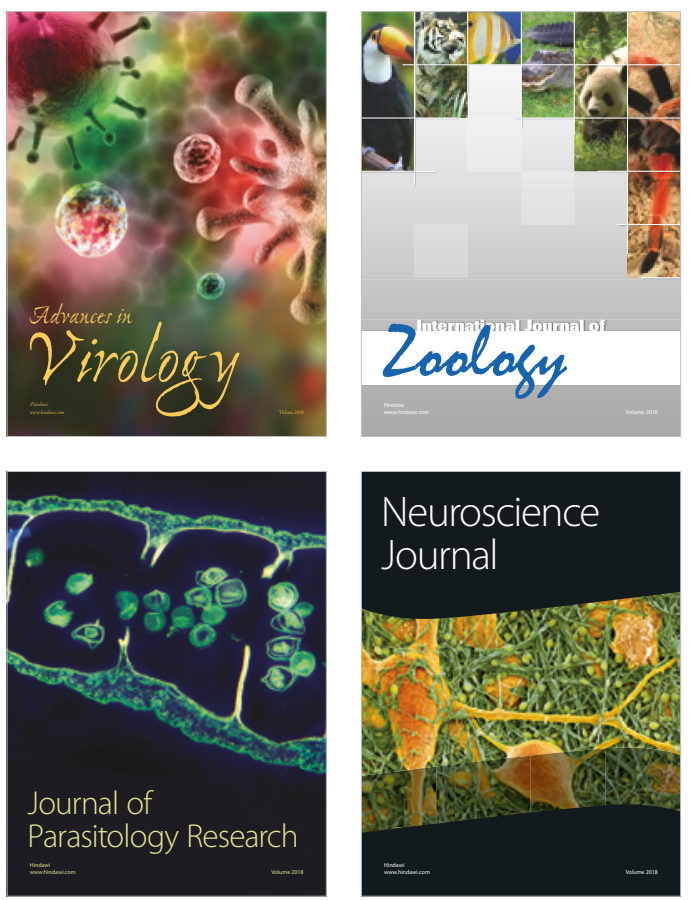
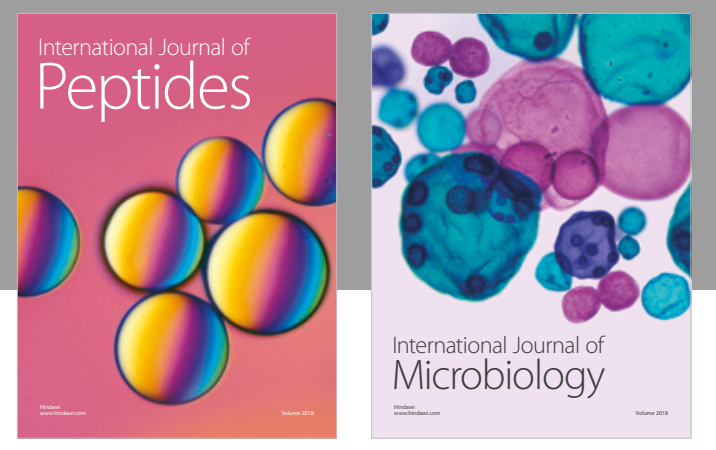

nternational Journal of Microbiology
Journal of
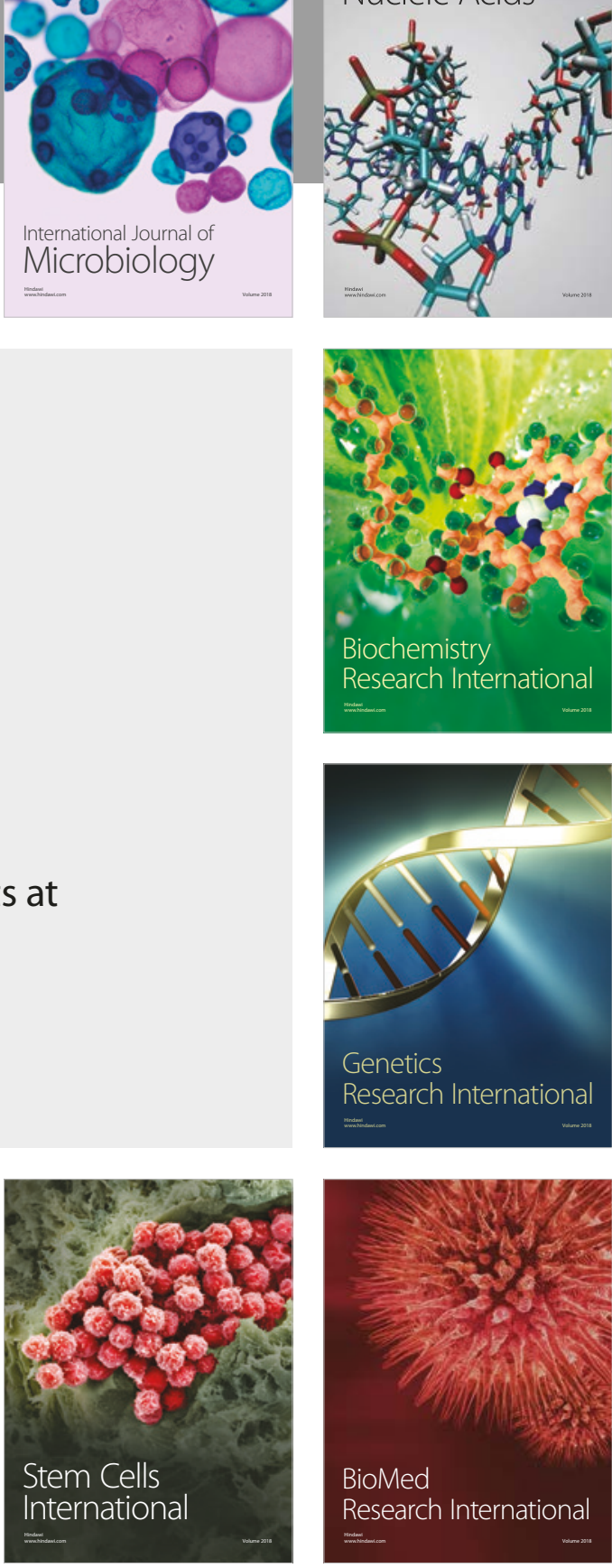
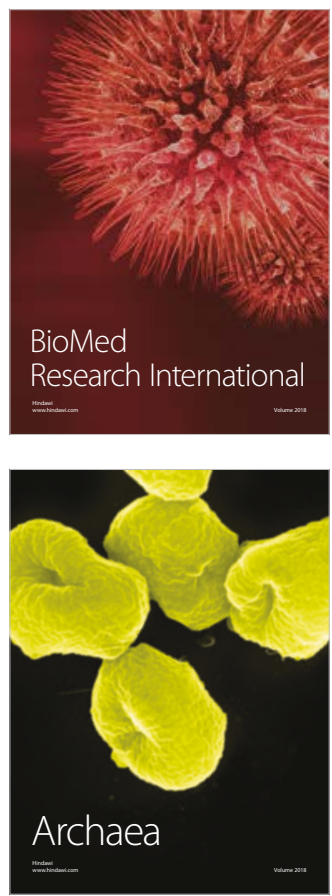